\title{
LOCALLY GRADED GROUPS WITH A NILPOTENCY CONDITION ON INFINITE SUBSETS
}

\author{
COSTANTINO DELIZIA, AKBAR RHEMTULLA and HOWARD SMITH
}

Dedicated to Professor B. H. Neumann on the occasion of his 90th birthday

(Received 18 March 1999; revised 1 May 2000)

Communicated by R. B. Howlett

\begin{abstract}
A group $G$ is locally graded if every finitely generated nontrivial subgroup of $G$ has a nontrivial finite image. Let $N(2, k)^{*}$ denote the class of groups in which every infinite subset contains a pair of elements that generate a nilpotent subgroup of class at most $k$. We show that if $G$ is a finitely generated locally graded $N(2, k)^{*}$-group, then there is a positive integer $c$ depending only on $k$ such that $G / Z_{c}(G)$ is finite.
\end{abstract}

2000 Mathematics subject classification: primary 20F19, $20 \mathrm{E} 26$.

Keywords and phrases: Infinite, nilpotent, groups, locally graded.

Let $k$ be a fixed positive integer. We denote by $N(2, k)$ the class of groups in which every 2-generator subgroup is nilpotent of class at most $k$, and by $N(2, k)^{*}$ the class of groups in which every infinite subset contains a pair of elements that generate a nilpotent subgroup of class at most $k$. The main result of [3] states that a finitely generated residually finite group $G$ belongs to $N(2,2)^{*}$ if and only if $G / Z_{2}(G)$ is finite. In [1] it was proved that if $G$ is finitely generated and soluble, then $G \in N(2, k)^{*}$ if and only if $G \in \mathfrak{F} N(2, k)$, where $\mathfrak{F}$ denotes the class of finite groups. It is remarked in [1] that if $G / Z_{k}(G)$ is finite, then $G \in \mathfrak{F} N(2, k)$ but that the converse is false for $k \geq 3$, even if $G$ is finitely generated and soluble of derived length three. The examples cited, which are due to Newman [8], are torsion-free nilpotent and hence residually finite. For $k=2$ there is the result from [2] that a finitely generated soluble group $G$ belongs to $N(2,2)^{*}$ if and only if $G / Z_{2}(G)$ is finite. Using some deep results of Zel'manov and Lubotzky and Mann, we are able to establish a theorem that provides

The second author thanks NSERC for partial financial support. The third author wishes to thank the Department of Mathematics at the University of Alberta for its fine hospitality.

(C) 2000 Australian Mathematical Society 0263-6115/2000\$A2.00+0.00 
what is essentially a generalization of each of the results mentioned above. First we recall that a group $G$ is locally graded if every finitely generated nontrivial subgroup of $G$ has a nontrivial finite image.

THEOREM 1. Let $G$ be a finitely generated locally graded group and suppose that $G \in N(2, k)^{*}$. Then there is a positive integer $c$ depending only on $k$ such that $G / Z_{c}(G)$ is finite.

Let us consider for the moment the special case where the group $G$ is finite-bynilpotent and satisfies the hypotheses of the theorem. Then $G$ has a finite normal subgroup $N$ with $G / N$ torsion-free nilpotent and, by [1, Lemma 2], $G / N \in N(2, k)$. A result of Zel'manov [11] now tells us that the class $t$ of $G / N$ is bounded in terms of $k$ only, and it follows that $\gamma_{t+1}(G)$ is finite and hence that $G / Z_{t}(G)$ is finite [5]. Thus in order to establish the theorem we need to show that the group in question is finite-by-nilpotent. In fact, it is the residually finite case that we need to deal with, and we shall see that the result follows quite easily once we have proved the following (where $\mathfrak{N}$ denotes the class of nilpotent groups).

PROPOSITION 1. Let $G$ be a finitely generated residually finite group in the class $N(2, k)^{*}$. Then $G \in \mathfrak{F N}$.

In order to establish Proposition 1 we require a number of preliminary results. But let us note at this point that there is a gap at the beginning of the proof of Theorem 1 in [3], in that the deduction that $G$ is hypercentre-by-finite relies on the assumption that $G$ is residually finite modulo its hypercentre, a fact that is not at all clear. It is possible to salvage the argument by means of techniques similar to those adopted in [3] (where Zel' manov's results are certainly not required), but we omit the details and proceed directly to the matter in hand.

In what follows $\mathfrak{N}_{k}$ denotes the class of nilpotent groups of class at most $k$.

LeMMA 1. Let $G \in N(2, k)^{*}$ and let $x, y \in G$. Then $\langle x\rangle^{(y)}$ is finitely generated.

PROOF. If $y$ is of finite order, then the result is clear, so assume $y$ has infinite order and consider the set $\left\{x, x y, x y^{2}, \ldots\right\}$. For some $i, j$ with $0 \leq i<j$ we have $\left\langle x y^{i}, x y^{j}\right\rangle \in \mathfrak{N}_{k}$ and hence $\left\langle x y^{i}, y^{m}\right\rangle \in \mathfrak{N}_{k}$, where $m=j-i$, so that $1=\left[x y^{i},{ }_{k} y^{m}\right]=$ $\left[x,{ }_{k} y^{m}\right]$. For $g \in G$ and $r \in \mathbb{N}$, an easy induction shows that $\left\langle\left[x,{ }_{i} g\right] ; 0 \leq i \leq r\right\rangle=$ $\left\langle x^{g^{i}} ; 0 \leq i \leq r\right\rangle,(\operatorname{read}[x, 0 g]=x)$, and it follows easily that $\langle x\rangle^{\langle y\rangle} \leq\left\langle x^{y^{i}} ;|i| \leq k m\right\rangle$, thus giving the result.

With the notation of [6], a group in the class $N(2, k)^{*}$ is therefore restrained, and repeated use of Corollary 4 of that paper gives the following (where $G^{(i)}$ denotes the $i$-th term of the derived series of $G$ ). 
COROLlaRY 1. Let $G \in N(2, k)^{*}$ and suppose that $G$ is finitely generated. Then $G^{(i)}$ is finitely generated for each positive integer $i$.

Next, suppose that $G$ is a finitely generated group in $N(2, k)^{*}$ and let $K$ be an arbitrary soluble image of $G$. Then $K$ is finite-by-nilpotent by [7, Theorem A], and hence finite-by-(nilpotent of $k$-bounded class), as we saw earlier. Thus the Hirsch length of $K$ is bounded by some integer that depends only on $k$ and the number of generators of $G$, and it follows that there is a positive integer $s=s(G)$ such that $G^{(j)} / G^{(j+1)}$ is finite for all $j \geq s$.

LEMMA 2. Let $G$ be a finitely generated group in the class $N(2, k)^{*}$ and let $H$ be an arbitrary subgroup of finite index in $G^{(s)}$, where $s=s(G)$. Then $H / H^{\prime}$ is finite.

PROOF. By Corollary 1 we know that $G^{(s)}$ is finitely generated, and it follows that $G^{(s)}$ is finite modulo the core $C$ of $H$ in $G$. If $C / C^{\prime}$ is finite, then so is $H / H^{\prime}$, so we may assume that $H$ is normal in $G$. Suppose for a contradiction that $H / H^{\prime}$ is infinite, and let $K / H^{\prime}$ denote its torsion subgroup. Choose $h \in H \backslash K, x \in G^{(s)}$ and consider the set $\left\{x, x h, x h^{2}, \ldots\right\}$. By hypothesis there are distinct integers $i, j$ such that $\left\langle x h^{i}, x h^{j}\right\rangle \in \mathfrak{N}_{k}$, and then $\left[h^{j-i},{ }_{k} x\right]=1$, so that $x$ has nontrivial centralizer $C_{1} / K$ in $H / K$. If this is not of finite index in $H / K$ then we may repeat the argument to obtain a series $K<C_{1}<C_{2} \leq H$, where $\left[C_{2}, x\right] \leq C_{1}$. Since $G^{(s)} / H$ is finite there is a positive integer $n$ such that $x^{n} \in H$, and $H / K$ is of course abelian. Thus modulo $K$ we have $1=\left[C_{2}, x^{n}\right]=\left[C_{2}, x\right]^{n}$; but $H / K$ is torsion-free and we have the contradiction $\left[C_{2}, x\right] \leq K$. We deduce that $H / C_{1}$ is finite and hence that $H=C_{1}$, that is, $[H, x] \leq K$. Since $x$ was arbitrary we now have $\left[H, G^{(s)}\right] \leq K$ and hence $G^{(s)} / K$ centre-by-finite, and it follows that $G^{(s+1)} K / K$ is finite. But $G^{(s)} / G^{(s+1)}$ is also finite, giving the contradiction that $H / K$ is finite. The lemma is therefore proved.

COROLLARY 2. Let $G$ and $s$ be as in Lemma 2 and suppose that $G$ is residually finite. If $H \in N(2, k)$ for some $H$ of finite index in $G^{(s)}$ then $G \in \mathfrak{F N}$.

PROOF. As before, $H$ is finitely generated. It is also residually finite and, by hypothesis, $k$-Engel. By [10, Theorem 2], therefore, $H$ is nilpotent. But $H / H^{\prime}$ is finite, by Lemma 2, and we deduce that $H$ is finite and hence that $G$ is finite-by-soluble and therefore finite-by-nilpotent [7].

Our next result, in conjunction with those above, will allow us to restrict our attention to groups $G$ that have a very special structure.

LEMMA 3. Let $L$ be a finitely generated residually finite group belonging to $N(2, k)^{*}$ and suppose that $H \notin N(2, k)$ for every subgroup $H$ of finite index in $L$. Then there 
exists a normal subgroup $G$ of finite index in $L$ such that $G=N\langle t\rangle$ for some normal subgroup $N$ of $G$ and element $t$ satisfying $\langle a t, b t\rangle \in \mathfrak{N}_{k}$ for all $a, b \in N$.

PROOF. Assume for a contradiction that $L$ has no such subgroup $G$. Then there exist elements $x_{0}, y_{0}$ of $L$ such that $\left\langle x_{0}, y_{0}\right\rangle \notin \mathfrak{N}_{k}$, and we may choose a normal subgroup $G_{1}$ of finite index in $L$ such that $\left\langle x_{0}, y_{0}\right\rangle G_{1} / G_{1} \notin \mathfrak{N}_{k}$. Put $w_{0}=y_{0}$.

If $\left\langle w_{0} x, w_{0} y\right\rangle \in \mathfrak{N}_{k}$ for all $x, y \in G_{1}$, then we may take $N=G_{1}$ and $w_{0}=t$ for a contradiction. Thus $\left\langle w_{0} x, w_{0} y\right\rangle \notin \mathfrak{N}_{k}$ for some $x_{1}, y_{1} \in G_{1}$, and there is a normal subgroup $G_{2}$ of finite index in $L$ and contained in $G_{1}$ such that $\left\langle w_{0} x_{1}, w_{0} y_{1}\right\rangle G_{2} / G_{2} \notin$ $\mathfrak{N}_{k}$. Put $w_{1}=w_{0} y_{1}$ and repeat the argument with $G_{1}$ replaced by $G_{2}$ and $w_{0}$ by $w_{1}$.

Continuing, we obtain a sequence of elements $z_{i}=w_{i-1} x_{i}$, where $w_{i}=w_{i-1} y_{i}$ for all $i \geq 1$, and a chain of subgroups $L>G_{1}>G_{2} \ldots$ with $\left\langle z_{i}, w_{i}\right\rangle G_{i+1} / G_{i+1} \notin \mathfrak{N}_{k}$ for each $i \geq 1$. Suppose $j>i \geq 1$. It is straightforward to show that $z_{j} \equiv w_{i}$ mod $G_{i+1}$, and thus we have $\left\langle z_{i}, z_{j}\right\rangle G_{i+1} / G_{i+1} \notin \mathfrak{N}_{k}$. It follows that $\left\langle z_{i}, z_{j}\right\rangle \notin \mathfrak{N}_{k}$ for all distinct $i, j$, contradicting the fact that $G \in N(2, k)^{*}$ and thus establishing the lemma.

In view of Lemma 2, Corollary 2 and Lemma 3, we may assume for the proof of Proposition 1 that $G$ has the following properties, say $(*)$ :

$G$ is finitely generated and residually finite, $G=N\langle t\rangle$ for some normal subgroup $N$ and element $t$ such that $\langle a t, b t\rangle \in \mathfrak{N}_{k}$ for all $a, b \in N$, and $H / H^{\prime}$ is finite for every subgroup $H$ of finite index in $G$.

LEMMA 4. If $G$ satisfies $(*)$, then every finite image of $G$ is nilpotent.

PROOF. For this we may assume that $G$ is itself finite. Let $x, y \in N$ be of order $p^{r}$, $q^{s}$ respectively, where $p, q$ are distinct primes. We show that $[x, y]=1$.

Write $t=t_{1} t_{2}$, where each of $t_{1}$ and $t_{2}$ is a power of $t, t_{1}$ is a $p^{\prime}$-element and $t_{2}$ is a $q^{\prime}$-element. For arbitrary $g \in G$ we have $\left\langle x^{g}, t\right\rangle=\left\langle x^{g} t, t\right\rangle \in \mathfrak{N}_{k}$. It follows that $\left[x^{g}, t_{1}\right]=1$ for all $g \in G$ and hence that $x$ centralizes $\left\langle t_{1}\right\rangle^{G}$. Thus $\langle x\rangle^{\left\langle t_{2} y\right\rangle}=\langle x\rangle^{\langle t y\rangle}$, which is a $p$-group since $\langle x, t y\rangle=\langle t y x, t y\rangle=\langle y x t, y t\rangle^{t^{-1}} \in \mathfrak{N}_{k}$. Since $\langle y, t\rangle \in \mathfrak{N}_{k}$ we have similarly that $\left[y, t_{2}\right]=1$, so there is a $q^{\prime}$-number $c$ such that $\left(t_{2} y\right)^{c}=y^{c}$, and it follows that $y \in\left\langle t_{2} y\right\rangle$ and hence that $\langle x\rangle^{\langle y\rangle}$ is a $p$-group. Similarly $\langle y\rangle^{\langle x\rangle}$ is a $q$-group, and we have $[x, y]=1$ as claimed.

It follows that $N$ is nilpotent and so $\gamma_{s+1}(N)=1$ for some integer $s$. Also $\langle x, t\rangle \in$ $\mathfrak{N}_{k}$ for all $x \in N$, and so for each $i \geq 1$ we get $\left[\gamma_{i}(N),{ }_{k} G\right] \leq\left[\gamma_{i}(N),{ }_{k}\langle t\rangle\right] \gamma_{i+1}(N) \leq$ $\gamma_{i+1}(N)$. Hence $\left[N,{ }_{k s} G\right]=1$ and the result follows.

If $G$ satisfies (*), then of course $G / G^{\prime}$ is finite, and Lemma 4 then implies that $G$ is residually a finite $\pi$-group for some finite set $\pi$ of primes. Thus $\bigcap_{p \in \pi} R_{p}=1$, where $R_{p}$ is the finite $p$-residual of $G$. Accordingly, it is only the case where $G$ is residually 
finite- $p$ that needs to be considered. Our final prerequisite requires the notion of a powerful $p$-group (for definitions and essential properties the reader is referred to $[4$, Chapter 2].

LEMMA 5. Let $G$ be a finite p-group that satisfies (*). Then there is an integer d depending only on $k$ and $p$, such that $\left(N^{d}\right)^{\prime}$ is a powerful subgroup of $N$.

ProOF. Let $A$ be a normal subgroup of $G$ contained in $N$ and let $a \in A, x \in N$ and write $z=x t$. Since $\langle a, z\rangle \in \mathfrak{N}_{k},\langle a\rangle^{\langle z\rangle}=\left\langle a^{z^{i}} ; 0 \leq i \leq k-1\right\rangle$ (much as in the proof of Lemma 1). Similarly $\langle a\rangle^{(t)}=\left\langle a^{t^{i}} ; 0 \leq i \leq k-1\right\rangle$. Also, since $\langle z, t\rangle \in \mathfrak{N}_{k}$ there is an integer $c=c(k)$ such that every element of $\langle z, t\rangle$ has the form $t^{u_{1}} z^{v_{1}} \cdots t^{u_{c}} z^{v_{c}}$, for integers $u_{i}, v_{i}$. Thus

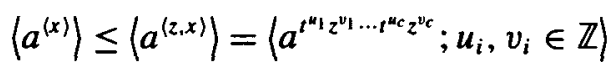

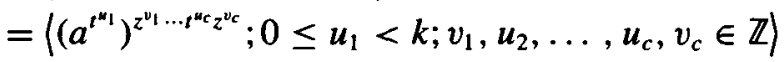

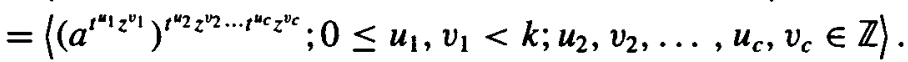

Inductively, we see that this is

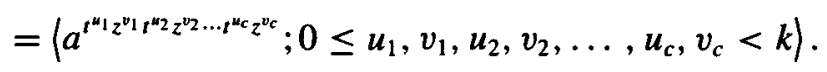

Define $M$ to be $A^{p} A^{\prime}$ if $p$ is odd, $A^{4} A^{\prime}$ if $p=2$. Then modulo $M,\langle a\rangle^{\langle z, x\rangle}$ has order bounded by some function of $p$ and $k$. It follows that $\left[a, x^{d}\right] \in M$ for some $d=d(p, k)$. But $x$ and $a$ were arbitrary, and we deduce that $\left[A, N^{d}\right] \leq M$.

With $J=N^{d}$ and $J^{\prime}=A$ we obtain $\gamma_{3}(J)=\left[J^{\prime}, J\right] \leq\left(J^{\prime}\right)^{p} \gamma_{4}(J)$ (respectively $\left.\left(J^{\prime}\right)^{4} \gamma_{4}(J)\right)$. Since $J$ is nilpotent it follows that $\left[J^{\prime}, J\right] \leq\left(J^{\prime}\right)^{p}$ (respectively $\left.\left(J^{\prime}\right)^{4}\right)$. Thus $J^{\prime}$ is powerfully embedded in $J$ and is therefore powerful and the lemma is proved.

PROOF OF Proposition 1. As we have seen, we may assume that $G$ satisfies (*) and that $G$ is residually a finite $p$-group for some prime $p$. Let $d$ be as in Lemma 5, so that $\left(N^{d}\right)^{\prime}$ is powerful in every finite quotient of $G$. Let $J$ denote the intersection of all subgroups $N^{d} K_{\lambda}$, where $K_{\lambda}$ is normal in $N$ and $N / K_{\lambda}$ is a finite $p$-group. By (*), $N$ has finite index in $G$ and is therefore finitely generated. So $N / J$ is a finitely generated residually finite group of exponent at most $d$ and hence finite, by [12] and [13]. It follows that $G / J^{\prime}$ is finite.

Write $P_{1}=J^{\prime}$, and for each positive integer $i$ let $P_{i+1}=\left(P_{i}\right)^{p}\left[P_{i}, J^{\prime}\right]$. By [4, Theorem 2.9] the rank of $J^{\prime} / P_{i}$ is precisely $d\left(J^{\prime}\right)$, the minimum number of elements required to generate $J^{\prime}$. With the notation of [4, Definition 6.2], the system $\left(P_{i}\right)$ therefore has finite rank, and [4, Theorem 6.3] then implies that $G$ is a linear group. Certainly $G$ does not contain a non-abelian free group, since such groups do not belong to $N(2, k)^{*}$, and so $G$ is soluble-by-finite [9]. Therefore $G$ is soluble by Lemma 4 , and the result follows from [7, Theorem $A]$. 
PROOF OF THEOREM 1. Let $G$ be as stated and let $R$ denote the finite residual of $G$. By the Proposition there is a finite normal subgroup $U / R$ of $G / R$ with $G / U$ nilpotent. By Lemma 1 above and repeated application of [6, Lemma 3], $U$ is finitely generated and hence $R$ is finitely generated. If $R=1$ then of course we are done, so assume $R$ is nontrivial, so that $R$ has a proper normal subgroup $S$ of finite index. Indeed, we may choose $S$ to be normal in $G$, and then $G / S$ is finite-by-nilpotent and therefore residually finite, contradicting the definition of $R$. The result follows.

\section{References}

[1] A. Abdollahi and B. Taeri, 'A condition on finitely generated soluble groups', Comm. Algebra, to appear.

[2] C. Delizia, 'Finitely generated soluble groups with a condition on infinite subsets', Istit. Lombardo Accad. Sci. Lett. Rend. A 128 (1994), 201-208.

[3] —_ 'On certain residually finite groups', Comm. Algebra 24 (1996), 3531-3535.

[4] J. D. Dixon, M. P. F. du Sautoy, A. Mann and D. Segal, Analytic pro-p groups, London Math. Soc. Lecture Note Series 157 (Cambridge Univ. Press, Cambridge, 1991).

[5] P. Hall, 'Finite-by-nilpotent groups', Proc. Cambridge Philos. Soc. 52 (1956), 611-616.

[6] Y. K. Kim and A. H. Rhemtulla, 'Weak maximality condition and polycyclic groups', Proc. Amer. Math. Soc. 123 (1995), 711-714.

[7] J. C. Lennox and J. Wiegold, 'Extensions of a problem of Paul Erdös on groups', J. Austral. Math. Soc. 31 (1981), 459-463.

[8] M. F. Newman, 'Some varieties of groups', J. Austral. Math. Soc. 16 (1973), 481-494.

[9] J. Tits, 'Free subgroups in linear groups', J. Algebra 20 (1972), 250-270.

[10] J. S. Wilson, 'Two-generator conditions for residually finite groups', Bull. London Math. Soc. 23 (1991), 239-248.

[11] E. I. Zel'manov, 'On some problems of group theory and Lie algebras', Mat. Sb. 180 (1989), 159-167. English translation: Math. USSR-Sb. 66 (1990), 159-168.

[12] - 'Solution of the restricted Burnside problem for groups of odd exponent', Izv. Akad. Nauk SSSR Ser. Mat. 54 (1990), 42-59. English translation: Math. USSR-Izv. 36 (1991), 41-60.

[13] _ 'Solution of the restricted Burnside problem for 2-groups', Mat. Sb. 182 (1991), 568-592.

Universitá di Napoli 'Federico II'

Dipartimento di Matematica e Applicazioni

Via Cintia - Monte S.Angelo, 80126 Napoli

Italy

e-mail: deliziac@matna2.dma.unina.it
Department of Mathematical Sciences

University of Alberta

Edmonton Alberta

Canada T6G 2G1

e-mail: akbar@malindi.math.ualberta.ca

\section{Department of Mathematics}

Bucknell University

Lewisburg PA 17837

USA

e-mail: howsmith@bucknell.edu 